\title{
Changeable Face Representations Suitable for Human Recognition
}

\author{
Hyunggu Lee, Chulhan Lee, Jeung-Yoon Choi, Jongsun Kim, and Jaihie Kim \\ School of Electrical and Electronic Engineering, Yonsei University, \\ Biometrics Engineering Research Center (BERC), Republic of Korea \\ \{lindakim, devices, jychoi,kjongss, jhkim\}@yonsei.ac.kr
}

\begin{abstract}
In order to resolve non-revocability of biometrics, changeable biometrics has been recently introduced. Changeable biometrics transforms an original biometric template into a changeable template in a non-invertible manner. The transformed changeable template does not reveal the original biometric template, so that secure concealment of biometric data is possible using a changeable transformation. Changeable biometrics has been applied to face recognition, and there are several changeable face recognition methods. However, previous changeable face transformations cannot provide face images that can be recognized by humans. Hence, 'human inspection', a particular property of face biometrics, cannot be provided by previous changeable face biometric methods.

In this paper, we propose a face image synthesis method which allows human inspection of changeable face templates. The proposed face synthesis method is based on subspace modeling of face space, and the face space is obtained by projection method such as principle component analysis (PCA) or independent component analysis (ICA). The face space is modeled by fuzzy C-means clustering and partition-based PCA. Using the proposed method, human-recognizable faces can be synthesized while providing inter-class discrimination and intra-class similarity.
\end{abstract}

Keywords: changeable face biometrics, non-invertibility, human inspection, face image synthesis, partition-based PCA.

\section{Introduction}

The human face is a key biometric characteristic for human recognition of personal identity. Current face recognition algorithms can handle large amounts of face biometric data very quickly. However, face recognition algorithms are not perfect. In many cases, face recognition algorithms are not as good as human inspection. Hence, the human ability of face recognition is still an important factor in recognition of a human face. Additionally, there are some examples where the human ability to recognize faces is an important issue, such as human inspection of the pictures of faces in a driver's license or a passport. In an ideal face recognition system, images of users are stored in a trusted database, and in that case, human inspection is possible using the face images of the database. 
However, storing user's face images can cause privacy violations. In order to resolve this problem, changeable biometrics has been introduced [1.

Ratha et al. 1] proposed a changeable face synthesis method based on morphing. Synthesizing face images by morphing allows human inspection. However, the original face image can be reconstructed when an attacker learns the morphing function. Besides the method of Ratha et al., there are several methods for generating changeable face biometrics [2] [3] 4]. A common point of these methods is that they cannot provide for human inspection.

Boult 2] proposed a transformation method for face recognition, in which a face feature is transformed via scaling and translation. Using robust distance measure, the transformed feature can provide improved performance, and the original biometric data cannot be inverted from their encrypted data, which is cryptographically secure. However, as mentioned, this transform does not allow human inspection.

Savvides et al. 3] proposed changeable biometrics for face recognition that uses minimum average correlation energy (MACE) filters and random kernels. However, the original face image can be recovered via deconvolution if the random kernel is known. Moreover, because the face is convolved with a random kernel, their method cannot provide for human inspection.

Teoh et al. 4] proposed a new authentication approach called BioHashing which combines user-specific tokenized random vectors with biometric feature vectors to generate a biometric code. The inner product between biometric feature vectors and a set of orthonormal random vectors are calculated and a biometric code is generated by using a predefined threshold. By thresholding, the original biometric can not be recovered from the biometric code. Again, however, their method does not provide for human inspection.

In this paper, we propose a subspace based changeable face synthesis method which allows human recognition of the generated faces. The organization of the paper is as follows. Section 2 briefly describes the method for generating changeable coefficient vectors from face images and our motivation for recognizable face synthesis. In Section 3, the subspace modeling and range fitting methods for face synthesis follow. Finally, results of our face synthesis and future work are discussed in Section 4 and Section 5, respectively.

\section{Changeable Coefficient Vector Generation}

A changeable face template can be generated by combining PCA and ICA coefficient vectors [5]. Two different face feature coefficient vectors, $\mathbf{P}$ and $\mathbf{I}$ are extracted from an input face image using PCA and ICA, and the two coefficient vectors are normalized as follows.

$$
\begin{aligned}
& \mathbf{p}=\mathbf{P} /|\mathbf{P}|=\left[\mathbf{p}_{1}, \mathbf{p}_{2}, \mathbf{p}_{3}, \ldots, \mathbf{p}_{N}\right], \\
& \mathbf{i}=\mathbf{I} /|\mathbf{I}|=\left[\mathbf{i}_{1}, \mathbf{i}_{2}, \mathbf{i}_{3}, \ldots, \mathbf{i}_{N}\right] .
\end{aligned}
$$

Then, the two normalized feature coefficient vectors, $\mathbf{p}$ and $\mathbf{i}$ are scrambled using two permutation matrices. 


$$
\mathbf{p}^{s}=\mathbf{S}_{I D}^{P C A}(\mathbf{p}), \quad \mathbf{i}^{s}=\mathbf{S}_{I D}^{I C A}(\mathbf{i})
$$

The permutation matrices, $\mathbf{S}_{I D}^{P C A}$ and $\mathbf{S}_{I D}^{I C A}$ are generated by a random number generator whose seed is the user's personal ID. Finally, a changeable face coefficient vector $\mathbf{c}$ is generated by addition of the scrambled coefficient vectors,

$$
\mathbf{c}=\mathbf{p}^{s}+\mathbf{i}^{s} .
$$

Even if an attacker knows the changeable face coefficient vector $\mathbf{c}$ and the scrambling rule, the original PCA or ICA coefficient vector can not be recovered because the changeable coefficient vector is generated by addition. Moreover, due to scrambling and addition, the changeable coefficient vector is not the same as either the PCA or the ICA coefficient vector, so that the changeable coefficient vector cannot represent a human-like image when the changeable coefficient vector is reprojected onto either the PCA or ICA basis. Fig. 1 shows some reprojected images using the PCA and ICA coefficient vectors and using the changeable coefficient vector. Fig. 1 (b) and (c) show reconstruction images using the original subspace coefficient vectors and corresponding subspace basis. Reconstructed images are rather degraded because we do not use the full number of basis vectors. The changeable coefficient vector can be directly reprojected using the PCA or ICA basis. Since the changeable coefficient vector differs from either the PCA or ICA coefficient vector, the reprojected images in Fig. 1 (d) and (e) cannot be recognized as human. Hence humans cannot inspect the resulting changeable face templates as to whether it is similar or not to a corresponding template in the database.

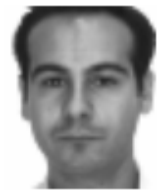

(a)

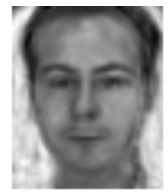

(b)

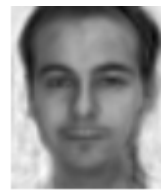

(c)

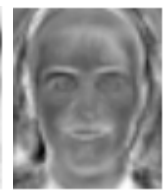

(d)

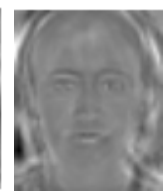

(e)

Fig. 1. Face generation using changeable coefficient vector (a): input face image, (b): reconstruction using the PCA coefficient vector, (c): reconstruction using the ICA coefficient vector, (d): reprojected image using changeable coefficient vector with the PCA basis, (e): reprojected image using changeable coefficient vector with the ICA basis

From the above discussion, it is clear that the changeable face template cannot yield a human face image. In fact, because the original face image cannot be stored in changeable biometrics, there is no specific face image that is associated with the changeable template. However, for human inspection, a new face image ought to be synthesized, and that synthesized face image should be generated from a changeable face template. In this paper, we propose a face synthesis method which can generate a new face image using a changeable coefficient vector from a modeled face subspace. In the following sections, the proposed face synthesis method is described more specifically. 


\section{Subspace Based Face Synthesis}

The proposed face image synthesis method is founded on face space modeling on a subspace such as the PCA or ICA coefficient vector space. As a subspace distribution modeling method, partition-based PCA follows fuzzy C-means clustering 6]. As shown in Fig. 2 (a) and (b), grouping is carried out by the clustering method, then PCA is applied to each cluster. Hereafter, this type of approach will be called 'partition-based $P C A$ '. However, due to differences of the modeled

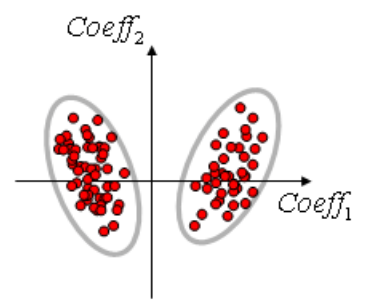

(a)

Partitioning: Fuzzy C-mean

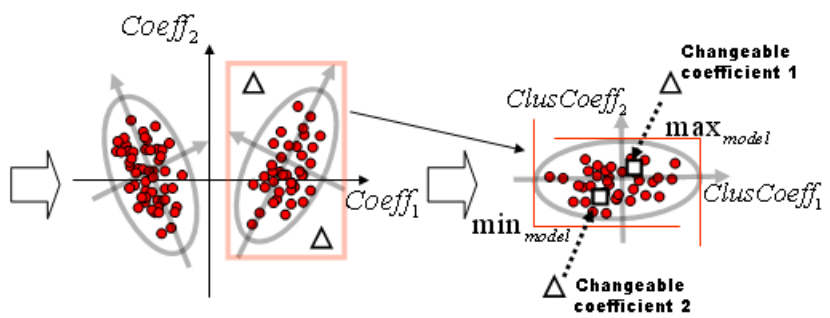

(b)

PCA to each cluster (c)

\section{Range fitting to \\ modeled distribution}

Fig. 2. Conceptual picture of face subspace modeling and range fitting for changeable coefficient vector (a): subspace is partitioned using clustering, (b): PCA is applied to each cluster, (c): range of changeable coefficient vector is fitted into range of coefficient vector of partition-based $P C A$

distributions between the face coefficient vector and the changeable coefficient vector, the changeable coefficient vector should be fitted within the modeled distribution of the face coefficient vector when synthesizing a face image. Before range fitting, the changeable coefficient vector is located outside of the modeled distribution of the face coefficient vector, e.g., the triangle points in Fig. 2 (c). After the range fitting process, the range fitted changeable coefficient vectors are located inside of the modeled distribution of the face coefficient vector, e.g., the square points in Fig. 2 (c). Because the face subspace coefficient vector, which exists inside of the modeled distribution of face coefficient vector, does represent a real face image, the range fitted changeable coefficient vector can also represent a face image that allows human inspection. Detail of the distribution modeling of face coefficient vector and the range fitting of the changeable coefficient vector into the distribution of the face coefficient vector will be covered in below.

\subsection{Face Distribution Modeling on the Subspace}

We use fuzzy C-means (FCM) method for subspace modeling, which is a soft clustering method. In order to update cluster prototypes, the overall information of data points are utilized with a weight which specifies the responsibility to cluster prototypes [6. After partitions are obtained from clustering, PCA is applied to each cluster. Local PCA [7] is a method similar to partition-based 
$P C A$. which iteratively updates partitions and PCA basis of the partitions using the reconstruction error. However, in our experiments, in order to utilize the local structure of initial clusters (FCM result), we use partition-based PCA as a non iterative version of local PCA.

\subsection{Local Surface Determination and Face Synthesis}

After FCM clustering, PCA is applied to each cluster in the subspace. Because PCA captures maximum variation of the distribution, the local surface of each subspace can be determined from the partition-based PCA components. For the $k$ th cluster of the subspace, $\mathbf{p}_{\text {cluster_ } k}^{i}$ is the $i$ th element of partition-based PCA coefficient vector $\mathbf{p}_{\text {cluster_k }}$. For face synthesis, $\mathbf{p}_{\text {cluster_k }}^{i}$ should be restricted with an upper and a lower limit, which can be selected using the mean $\mathbf{m}_{\text {cluster_k }}^{i}$ and standard deviation $\sigma_{\text {cluster_k }}^{i}$ of $\mathbf{p}_{\text {cluster_k }}^{i}$.

$$
\begin{aligned}
& \min _{\text {Cluster_k}}^{i} \leq \mathbf{p}_{\text {Cluster_k }}^{i} \leq \max _{\text {Cluster_k }}^{i}, \\
& \text { where }\left(\begin{array}{l}
\min _{\text {Cluster_k}}^{i}=\mathbf{m}_{\text {Cluster_k}}^{i}-\alpha \sigma_{\text {Cluster_ } k}^{i} \\
\max _{\text {Cluster_k }}^{i}=\mathbf{m}_{\text {Cluster_k }}^{i}+\alpha \sigma_{\text {Cluster_k }}^{i} .
\end{array}\right.
\end{aligned}
$$

As a result, the local surface of the $k$ th cluster can be determined by $\min _{\text {cluster_ } k}^{i}$ and $\max _{\text {cluster_k }}^{i}$ of $\mathbf{p}_{\text {cluster_k}}^{i}$ with scale factor $\alpha$ which controls the width of the modeled local surface. For face synthesis, partition-based PCA coefficient vector $\mathbf{p}_{\text {cluster_ } k}$ should be selected within the local surface of the $k$ th cluster. The selected $\mathbf{p}_{\text {cluster_k }}$ is associated with one synthesized face in the corresponding subspace, and face images can be generated as follows. The synthesized face coefficient vector $\lambda_{\text {syn }}$ is the result of reprojecting $\mathbf{p}_{\text {cluster_k }}$ into the corresponding partitionbased PCA basis $\boldsymbol{\Phi}_{\text {cluster_k }}$.

$$
\lambda_{\text {syn }}=\boldsymbol{\Phi}_{\text {cluster_ } k} \mathbf{p}_{\text {cluster_ } k} .
$$

After the synthesized face coefficient vector $\lambda_{\text {syn }}$ is obtained, the synthesized face image $\mathbf{x}_{\text {syn }}$ can be generated by reprojection with the corresponding subspace basis $\Psi$.

$$
\mathbf{x}_{\text {syn }}=\mathbf{\Psi} \lambda_{\text {syn }} .
$$

Hence, a face image can be synthesized using $\mathbf{p}_{\text {cluster_ } k}$. However, a changeable face image is synthesized from a changeable coefficient vector $\mathbf{c}$. Therefore, the changeable coefficient vector $\mathbf{c}$ should be converted accordingly. The conversion of $\mathbf{c}$ is done by range fitting; the range fitted changeable coefficient vector is $\mathbf{y}_{\text {cluster_k}}$. To do this, the range of the changeable coefficient vector should be determined. After range determination, the changeable coefficient vector is fitted within the distribution of partition-based $P C A$ coefficient vector $\mathbf{p}_{\text {cluster_k }}$. Then $\mathbf{y}_{\text {cluster_k }}$ is able to represent a face image that is recognizable by a human using reprojection as follows:

$$
\mathbf{x}_{\text {syn }}=\boldsymbol{\Psi} \boldsymbol{\Phi}_{\text {cluster_k }} \mathbf{y}_{\text {cluster_k }} .
$$




\subsection{Determining the Range of the Changeable Coefficient Vector}

Because a changeable coefficient vector is a mixed feature of two different subspace coefficient vectors (PCA and ICA), the distribution of the changeable coefficient vector is unrelated to the original two subspace coefficient vectors. However, if we assume that the original two coefficient vectors are statistically independent, then because the changeable coefficient vector $\mathbf{c}$ is no more than the addition of PCA and ICA coefficient vectors, the range of $\mathbf{c}$ can be determined from the ranges of the PCA and ICA coefficient vectors. The range of $\mathbf{p}_{i}$, the $i$ th element of PCA coefficient vector $\mathbf{p}$, can be obtained from the training data, which exists between a lower limit $\min _{p c a}^{i}$ and upper limit $\max _{p c a}^{i}$. In a similar way, the range of $\mathbf{i}_{i}$, the $i$ th element of ICA coefficient vector $\mathbf{i}$, can be obtained. Because the scrambling rule, i.e., $\mathbf{S}_{I D}^{P C A}$ and $\mathbf{S}_{I D}^{I C A}$, are stored for changeable coefficient vector generation, they can also be used for range determination of the changeable coefficient vector. Hence, scrambling can be ignored for notational simplification. Then, $\mathbf{c}_{i}$ becomes an addition of $\mathbf{p}_{i}$ and $\mathbf{i}_{i}$. Using the independence assumption between $\mathbf{p}_{i}$ and $\mathbf{i}_{i}$, the range of $\mathbf{c}_{i}, i$ th element of changeable coefficient vector $\mathbf{c}$, can be inferred by examining the range of $\mathbf{p}_{i}$ and $\mathbf{i}_{i}$ as follows:

$$
\begin{aligned}
& \min _{c}^{i} \leq \mathbf{c}_{i} \leq \max _{c}^{i}, \\
& \text { where }\left(\begin{array}{l}
\min _{c}^{i}=\min _{p c a}^{i}+\min _{i c a}^{i} \\
\max _{c}^{i}=\max _{p c a}^{i}+\max _{i c a}^{i}
\end{array} .\right.
\end{aligned}
$$

\subsection{Range Fitting of Changeable Coefficient Vector into partition-based PCA Coefficient Vector}

The range of the changeable coefficient vector is then fitted into the range of the partition-based PCA coefficient vector. Using the ranges as determined in previous subsection, the changeable coefficient vector $\mathbf{c}$ is converted to be in the range of partition-based $P C A$ coefficient vector $\mathbf{p}_{\text {cluster_k }}$. Because there is no restrictions on selection of cluster partition $k, k$ can be selected freely for each person, and the converted coefficient vector is represented by $\mathbf{y}_{\text {cluster_ }} k$.

$$
\begin{gathered}
\mathbf{y}_{\text {Cluster } \_k}^{i}=\left(\frac{\mathbf{c}_{i}-\min _{c}^{i}}{\operatorname{maxa}_{c}^{i}-\min _{c}^{i}}\right) \times\left(\max _{\text {Cluster } \_k}^{i}-\min _{\text {Cluster_k }}^{i}\right) \\
+\min _{\text {Cluster_k }}^{i} .
\end{gathered}
$$

As a result, the synthesized face image $\mathbf{x}_{\text {syn }}$ can be reprojected from $\mathbf{y}_{\text {cluster_ } k}$ using the corresponding basis of partition-based PCA and the basis of the subspace.

$$
\mathbf{x}_{\text {syn }}=\boldsymbol{\Psi} \boldsymbol{\Phi}_{\text {cluster_k } k} \mathbf{y}_{\text {cluster_ } k} .
$$

\section{Experiment and Result}

In our experiment, 492 frontal facial images from the AR database 8 are used. Images with occlusion and illumination changes are excluded, for a total of 6 
different images per subject. The dimension of PCA and ICA spaces is set to 100. For face space modeling, FCM is applied to the face coefficient vector space. In our experiments, two distinct clusters are formed for each coefficient vector space. When generating a changeable coefficient vector, different scrambling rules are applied to each person. Performance can be evaluated in two aspects. One is the EER using the generated changeable coefficient vector $\mathbf{c}$, and the other is the EER using the range fitted coefficient vector $\mathbf{y}_{\text {cluster }} k$. The EER of the changeable coefficient vector $\mathbf{c}$ can be used to evaluate the performance of the changeable face recognition system, and the EER of the range fitted coefficient vector $\mathbf{y}_{\text {cluster_k }}$ can be used for quantitative evaluation of human inspectibility. As Table 1 shows, due to different scrambling of changeable coefficient vectors for each person, the EER of the changeable coefficient vector is very low. The EER of $\mathbf{y}_{\text {cluster_k }}$ fitted to ICA clusters is similar to the EER using ICA coefficient vectors, and the EER of $\mathbf{y}_{\text {cluster_k }}$ fitted to PCA clusters is similar to the EER using PCA coefficient vectors. This result is due to the range fitting process. During range fitting, whatever the value of the changeable coefficient vector $\mathbf{c}$, the range fitted coefficient vector $\mathbf{y}_{\text {cluster }} k$ is located within the local surface of the modeled subspace cluster. Hence, EER of the range fitted coefficient vector is similar to the EER of the corresponding subspace coefficient vector. This implies that inter-class discrimination and intra-class similarity of the original input faces is preserved in the synthesized changeable faces.

Table 1. Performance of changeable coefficient vector and partition-based PCA coefficient vectors of modeled clusters

\begin{tabular}{|c|c|c|c|c|}
\hline $\begin{array}{c}\text { PCA } \\
\text { coefficient } \\
\text { vector }\end{array}$ & $\begin{array}{c}\text { ICA } \\
\text { coefficient } \\
\text { vector }\end{array}$ & $\begin{array}{c}\text { Changeable } \\
\text { coefficient } \\
\text { vector }\end{array}$ & $\begin{array}{c}\text { Range fitted } \\
\text { coefficient } \\
\text { vector } \\
\text { - ICA cluster }\end{array}$ & $\begin{array}{c}\text { Range fitted } \\
\text { coefficient } \\
\text { vector } \\
\text { - PCA cluster }\end{array}$ \\
\hline $15.03 \%$ & $12.6 \%$ & $0.02 \%$ & $13.83 \%$ & $14.88 \%$ \\
\hline
\end{tabular}

Fig. 3 shows the result of changeable face image synthesis. Because two clusters are formed for each subspace, two types of face images can be synthesized from each subspace. Fig. 3 (d) and (e) show synthesized face images from ICA clusters, and Fig. 3 (h) and (i) show synthesized images from PCA clusters. In both subspaces, using changeable coefficient vectors, synthesized images from different clusters show distinct characteristics, such as gender. Using the proposed face synthesis method, we can represent any person as a different person of either gender. From Fig. 3, we can discern the distinctiveness of the synthesized face image for each person. Hence, the above result shows inter-class discrimination of the proposed face synthesis method. However, synthesized faces from the same person's images should show similarity between synthesized faces. This intra-class similarity can be shown in Fig. 4. Input faces of Fig. 4 (a) contain expressional variation. Fig. 4 (b) is a result of synthesized images from ICA cluster 1, and Fig 4 (c) is a result of synthesized images from ICA cluster 2. For 


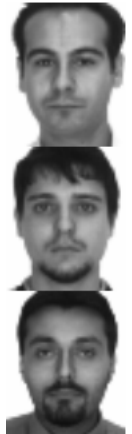

(a)

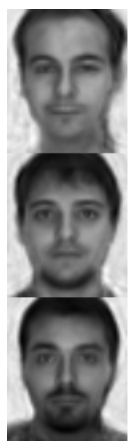

(b)

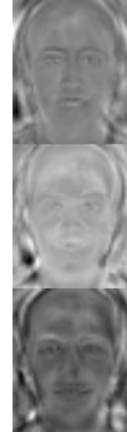

(c)

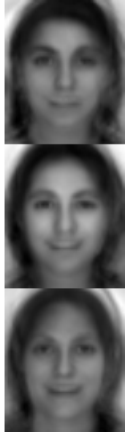

(d)

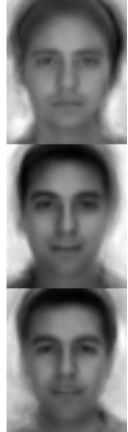

(e)

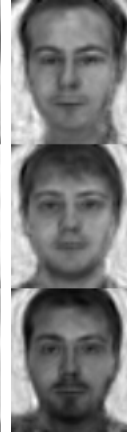

(f)

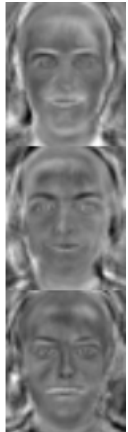

(g)

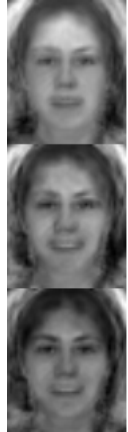

(h)

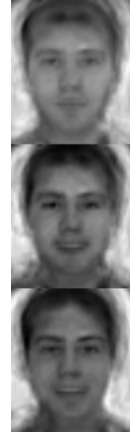

(i)

Fig. 3. Synthesized changeable faces (a):input image, (b):reconstructed image by ICA basis, (c):direct reprojection of changeable coefficient vector using ICA basis, (d):synthesized image from cluster 1 of ICA, (e):synthesized image from cluster 2 of ICA, (f):reconstructed image by PCA basis, (g): direct reprojection of changeable coefficient vector using PCA basis, (h):synthesized image from cluster 1 of PCA, (i):synthesized image from cluster 2 of PCA

Fig. 4 (b) and (c), because images of the same row are synthesized from the same person's input images, images within a row are similar each other. However, images of different rows are synthesized from images of different person, and any two images between two different rows are not similar. From Fig. 3 and Fig. 4, it can be shown that synthesized face images can be used to substitute the original face image. In other words, with respect to inspectability, the proposed face synthesis method shows intra-class similarity while providing inter-class discrimination. The similarity between the EER using the original subspace coefficient vector and the EER using the changeable coefficient vector of Table 1 supports the above results.

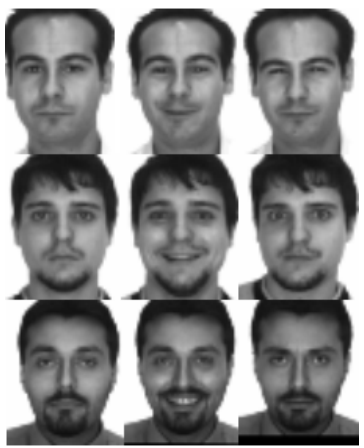

(a)

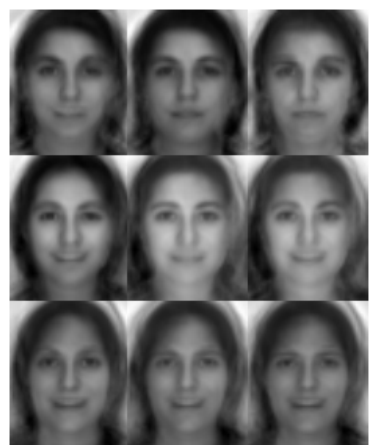

(b)

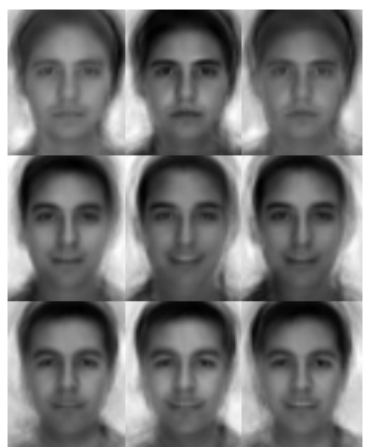

(c)

Fig. 4. Synthesized changeable faces of two ICA clusters (a): input faces, (b): synthesized faces from ICA cluster 1, (c): synthesized faces from ICA cluster 2 


\section{Conclusion and Future Works}

The proposed face synthesis method for changeable face biometrics can generate face images which allow human recognition. The quality of the synthesized face image depends on the accuracy of the subspace modeling method and the compactness of the subspace. From the point of view of human inspectability, face image synthesis using ICA cluster modeling is better than PCA cluster modeling. The quality of the synthesized face image also can be improved by using a more accurate subspace modeling method. In this work, face subspace modeling is accomplished by a simple clustering method (FCM) and partition based PCA. However, the face subspace can be modeled by more accurate clustering methods such as Gaussian Mixture Models (GMM) or Gustafson-Kessel clustering, etc. In future works, such subspace modeling methods may be tested for changeable face synthesis.

\section{Acknowledgements}

This work was supported by the Korea Science and Engineering Foundation (KOSEF) through the Biometrics Engineering Research Center (BERC) at Yonsei University

\section{References}

1. Ratha, N.K., Connell, J.H., Bolle, R.M.: Enhancing security and privacy in biometrics-based authentication systems. IBM Systems Journal. 40, 614-634 (2001)

2. Boult, T.: Robust Distance Measures for Face-recognition supporting revocable biometrics token. In: 7th International Conference Automatic Face and Gesture Recognition, pp. 560-566 (2006)

3. Savvides, M., Vijaya Kumar, B.V.K., Khosla, P.K.: Cancelable Biometric Filters for Face Recognition. In: Proc. of the 17th International Conference on Pattern Recognition (ICPR 2004), vol. 3, pp. 922-925 (2004)

4. Teoh, A.B.J., Ngo, D.C.L., Goh, A.: BioHashing: two factor authentication featuring fingerprint data and tokenised random number. Pattern Recognition 37, 2245-2255 (2004)

5. Jeong, M.Y., Lee, C.H., Kim, J.S., Choi, J.Y., Toh, K.A., Kim, J.H.: Changeable biometrics for appearance based face recognition. In: The Biometric Consortium Conference, Baltimore convention center, Baltimore, MD, USA, September 19th21th, 2006 (2006)

6. Bezdek, J.C.: Pattern Recognition with Fuzzy Objective Function Algorithms. Plenum Press, New York (1981)

7. Kambhatla, N., Leen, T.K.: Dimension Reduction by Local Principal Component Analysis. Neural Computation 9, 1493-1516 (1997)

8. Martinez, A.M., Benavente, R.: The AR Face Database. CVC Technical Report. \#24 (1998) 